\section{An interactive program to build a computer library of literature references}

\section{TIM B. HUNTER and GEORGE W. SEELEY University of Arizona, Tucson, Arizona}

Hunter (1983) described a computer program that allows the user to build and search through a library of literature references. The present article describes an extension and refinement of that program. Our goal was to design an easy-to-use, general-purpose system for storing literature citations.

Two programs, REFERSET and REFERENCE, were written using the BASIC 11 language on the Digital Equipment Corporation VAX $11 / 780$ computer. BASIC was chosen as the programming language because of its ready availability, ease of use, adaptability to microsystems, and user-friendly logic.

REFERSET. REFERSET initializes the files used by REFERENCE (Table 1). It needs to be run only once. After the files are established, storage of up to 2,500 reference listings is allowed.

REFERENCE. REFERENCE was written so that it can be used by someone relatively unfamiliar with programming. It permits the addition of new reference entries, the deletion of unwanted entries, and the use of a variety of data search and retrieval methods. There are three types of reference searches possible: an author search, a keyword search, and a search through the article listing itself.

Figure 1 shows a typical reference entry. The program asks the user for the following items during the addition of a reference: (1) author(s) (up to 255 characters), (2) article or book listing (up to 255 characters), and (3) keywords (up to 100 characters). Entered data are immediately redisplayed to allow for corrections. All data entry, deletion, correction, and searching can be performed by someone unfamiliar with programming, because only a few minutes of practice at the terminal are needed to understand the program operation.

The author-search routine uses up to five names in a loose manner; that is, any reference having any one of the requested authors is displayed on the CRT screen. The keyword search routines use up to five separate keyword phrases in either a loose or restrictive manner. A restrictive search retrieves only those references that match all the selected keyword phrases. For example, if a reference entry has the keyword phrases "ROC curve, image perception, detection parameters," a loose search outputs all references matching any one

This work was partially funded by NIH Grant CA23417. Tim B. Hunter is with the Department of Radiology, Arizona Health Sciences Center, and George W. Seeley is with the Optical Sciences Center, both at the University of Arizona, Tucson, Arizona 85721.
Table 1

Files Created by REFERSET for Use by the Program REFERENCE

\begin{tabular}{llrr}
\hline $\begin{array}{c}\text { Information } \\
\text { Contained }\end{array}$ & \multicolumn{1}{c}{ File Name } & $\begin{array}{c}\text { Character } \\
\text { Width }\end{array}$ & $\begin{array}{c}\text { Size } \\
\text { (in Bytes) }\end{array}$ \\
\hline Author & REFERENCE.AUT & 255 & 637,952 \\
Reference Entry & REFERENCE.REF & 255 & 637,952 \\
Keywords & REFERENCE.KEY & 100 & 250,368 \\
Flag & REFERENCE.CNT & 1 & 10,240 \\
Pointer & REFERENCE.PNT & 4 & 512 \\
Total & & 615 & $1,537,024$ \\
\hline
\end{tabular}

Note-Program-REFERENCE; size (in bytes)-12,288; up to 2,500 reference entries allowed.

of these phrases. A restrictive search outputs only those references whose keyword phrases match exactly all three of these phrases. Searching by means of the reference entry is done in a loose manner that uses up to three search parameters.

The program also assigns a number to each reference entry. This number is useful in two ways. First, copies of the reprints can be filed in consecutive order according to these assigned numbers. Thus, when a search of

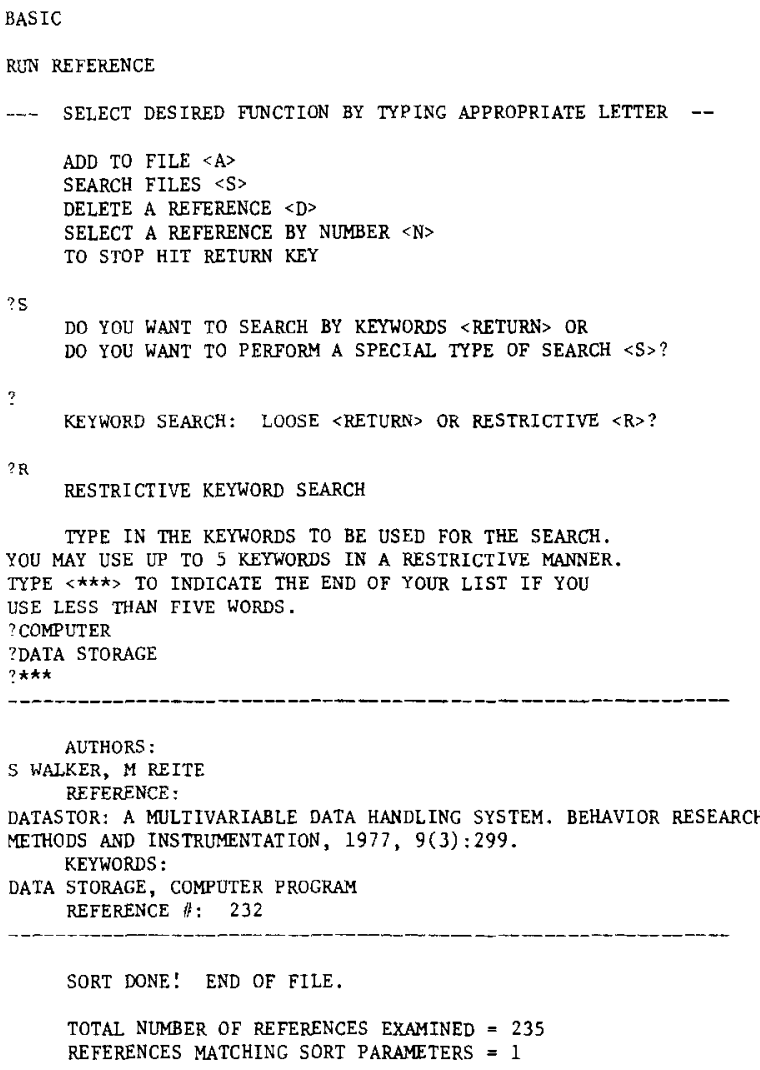

Figure 1. Sample printout from a restrictive search of the key word plurases "Data storage, computer program." 
the reference data base yields a list of articles, they can be readily accessed in their storage locations by using their reference numbers. Second, the program user can alter any previously entered data merely by entering the reference number for a particular reference entry.

For best effect, all data should be entered in a consistent manner. A standard style for entering authors names, reference listing, and keywords should be decided upon by the user prior to the first data entry. The reason for this is the limitation of the sort. For example, if one of the authors' names, "Tim B. Hunter," is entered as (1) Hunter, TB, or (2) Hunter, T.B., searching by "Hunter, T.B." will pick up the second entry only. In designing the data entry protocol, spelling, spaces, periods, and commas must be taken into account. Sloppily entered data will result in a decreased output during retrieval.

A pointer variable in the program keeps track of the next available location for data entry. For example, if a reference is deleted, its space will be filled upon the next entry prior to the opening up of any new space. In any other case, the references are stored sequentially. Thus, no space is left unused.

All choices offered to the user during program execution are written so that the most frequent ones are defaulted and indicated by striking the RETURN (CR) button. Unusual requests, such as deleting an entry, must be specifically chosen by the user. This is to prevent common, inadvertant mistakes.

Limitations and Suggestions. As presently written, the program runs on a system that has a CRT display in parallel with a printer (Hunter, 1983). Information displayed on the screen can be printed or not by turning on the printer at one's discretion. The program is written as simply as possible and does not allow data to be sent to a remote printer or display. Since most printers are slow and do not have buffers, 300 baud is the recommended speed for printing. If the program is to be run faster than 1,200 baud, the code must be modified to halt after the CRT screen is filled and then continue when the user gives a command [such as RETURN (CR) or a control character] to see the screen filled again. This modification is necessary to prevent entries from flashing by on the screen at too rapid a rate for evaluation.

Availability and Transportability. A complete listing of the program, with comments and a short documentation, is available by sending a self-addressed $9.5 \mathrm{x}$ $12.5 \mathrm{in}$. envelope, with enough postage for $7 \mathrm{oz}$., and $\$ 5.00$ for the cost of the printed materials to: Dr. Tim Hunter, Department of Radiology, Arizona Health Sciences Center, University of Arizona, Tucson, Arizona 85724. REFERENCE uses Digital Equipment Corporation (DEC) BASIC 11 as outlined in the BASIC 11 Language Reference Manual (Digital Equipment Corporation, 1976). Other versions of BASIC will differ somewhat, and the program will have to be translated. However, once the underlying logic is understood by the user, it can be incorporated into other systems and modified to the user's own particular needs.

\section{REFERENCES}

Digital Equipment Corponation. BASIC 11 language reference manual. Maynard, Mass: Author, 1976.

HunTER, T. B. Simple interactive computer program to store literature references. American Journal of Roentgenology, 1983, 140, 169.

(Manuscript accepted for publication May 6, 1983.) 